\title{
Abnormal expression of key genes and proteins in the canonical Wnt/ $\beta$-catenin pathway of articular cartilage in a rat model of exercise-induced osteoarthritis
}

\author{
SHEN-SHEN LIU ${ }^{1}$, PU ZHOU ${ }^{1}$ and YANQIU ZHANG ${ }^{2}$ \\ ${ }^{1}$ College of Physical Education, Langfang Teachers University, Langfang, Hebei 065000; \\ ${ }^{2}$ Department of Physical Education, Xi'an Shiyou University, Xi'an, Shaanxi 710065, P.R. China
}

Received February 27, 2015; Accepted December 23, 2015

DOI: $10.3892 / \mathrm{mmr} .2016 .4798$

\begin{abstract}
To investigate the molecular pathogenesis of the canonical Wnt/ $\beta$-catenin pathway in exercise-induced osteoarthritis (OA), 30 male healthy Sprague Dawley rats were divided into three groups (control, normal exercise-induced $\mathrm{OA}$ and injured exercise-induced OA groups) in order to establish the exercise-induced OA rat model. The mRNA and protein expression levels of Runx-2, BMP-2, Ctnnb1, Sox-9, collagen II, Mmp-13, Wnt-3a and $\beta$-catenin in chondrocytes were detected by reverse transcription-quantitative polymerase chain reaction, western blotting and immunohistochemical staining. The mRNA levels of Runx-2, BMP-2 and Ctnnb1 were upregulated in the normal exercise-induced OA and injured exercise-induced OA groups; while Runx-2 and BMP-2 were upregulated in the injured exercise-induced OA group when compared with the normal exercise-induced OA group. The protein levels of Mmp-13, Wnt-3a and $\beta$-catenin were increased and collagen II was reduced in the normal exercise-induced $\mathrm{OA}$ and injured exercise-induced $\mathrm{OA}$ groups. Ctnnb1, Wnt-3a and $\beta$-catenin, which are key genes and proteins in the canonical $\mathrm{Wnt} / \beta$-catenin pathway, were abnormally expressed in chondrocytes of the exercise-induced OA rat model. Ctnnb1, $\beta$-catenin and Wnt-3a were suggested to participate in the pathogenesis of exercise-induced OA by abnormally activating the $\mathrm{Wnt} / \beta$-catenin pathway during physical exercise due to excessive pressure. The results of the present study may provide an improved understanding of the pathogenesis of exercise-induced OA.
\end{abstract}

Correspondence to: Mr. Shen-Shen Liu, College of Physical Education, Langfang Teachers University, 100 Aimin West Road, Langfang, Hebei 065000, P.R. China

E-mail: liuss1981@126.com

Key words: exercise-induced osteoarthritis, Wnt/ $\beta$-catenin pathway, rat model, molecular pathogenesis

\section{Introduction}

Osteoarthritis (OA) is a type of chronic, progressive and common clinical articular cartilage disease. It is also termed hypertrophic arthritis or degenerative arthritis; the pathological characteristics of the disease include degeneration of articular cartilage, sclerosis of subcartilaginous bone, reactive hyperplasia of subcartilaginous bone and osteophyte formation (1). Primary OA predominantly occurs in middle-aged and elderly people, and the disease results from the disturbance of catabolism and anabolism in chondrocytes and the extracellular matrix with the common effects of biological and mechanical factors (2). The practice of high-loaded and antagonistic types of physical exercise has been increasing, such that the young population are inflicting excessive pressure on their knee joints, which may lead to serious knee joint injuries (3). Excessive wear and injury of articular cartilage, which is predominantly mediated by mechanical factors, may result in the aging and degeneration of articular cartilage, which gives rise to pain, lesions and dysfunctions of the joints $(4,5)$. At present, the pathogenesis of exercise-induced OA in the area of sports medicine remains unknown, therefore, it is of significant importance to investigate its pathogenesis.

Exercise-induced OA is a type of OA, where the inflammatory signaling pathway-associated cytokines, including interleukin $1 \beta$, tumor necrosis factor $\alpha$, serve an important role in the disturbance of catabolism and anabolism in chondrocytes and the extracellular matrix. It is the inflammatory cytokines that alter the metabolism of chondrocytes, the histological structures of the extracellular matrix and the activation of matrix metalloproteinase, which lead to disturbance in the synthesis and degradation of cartilage matrix, thus resulting in the development of OA $(6,7)$. However, how the cytokines transduce signaling into the chondrocytes and regulate the inflammatory genes remains unclear.

$\beta$-catenin is a multifunctional protein in the cytoplasm, and a key molecule in the Wnt signaling pathway for regulating gene transcription (8). The canonical Wnt/ $\beta$-catenin pathway serves a key role in the process of formation and differentiation of chondrocytes and the metabolism in the extracellular matrix. The inactivation of $\beta$-catenin may lead to the differentiation of chondrocytes and cell death (9). However, the 
association between the canonical Wnt/ $\beta$-catenin pathway and the pathogenesis of exercise-induced OA remains to be fully elucidated.

The present study aimed to investigate the molecular pathogenesis of the canonical Wnt/ $\beta$-catenin pathway in exercise-induced $\mathrm{OA}$ by establishing a exercise-induced OA rat model, and detecting the key genes and proteins of the canonical Wnt/ $\beta$-catenin pathway by reverse transcription-quantitative polymerase chain reaction (RT-qPCR), western blotting and immunohistochemical staining.

\section{Materials and methods}

Experimental animals. A total of 30 healthy male Sprague-Dawley rats (age, 5-6 weeks; weight, 160-230 g) were were provided by the Laboratory Animal Center of Xi'an Jiaotong University Health Science Center (Xi'an, China) for use in the present study. The rats were raised in individual cages and had ad libitum access to food and water. The feed-stuff was provided by the Laboratory Animal Center of Xi'an Jiaotong University Health Science Center, according to national standards of rodent animal feed. Indoor temperature $25 \pm 3^{\circ} \mathrm{C}$, relative humidity $55-75 \%$. The present study was approved by the ethics committee of Langfang Teachers University (Langfang, China).

\section{Experimental methods}

Animal groups. Following feeding for 5 days in order to adapt to the environment, the 30 Sprague-Dawley rats were randomly divided into three groups ( $\mathrm{n}=10$ /group), the control, normal exercise-induced $\mathrm{OA}$ and injured exercise-induced $\mathrm{OA}$ groups. The rats in the control group did not undergo operation nor training.

Establishment of the exercise-induced OA rat model. In each rat of the injured exercise-induced OA model group, the anterior and posterior cruciate ligaments of their right knee joints were cut. Firstly, the rats were anesthetized by intraperitoneal injection with $10 \%$ chloral hydrate $(0.3 \mathrm{ml} / 100 \mathrm{~g}$; Sinopharm Chemical Reagent Co., Ltd., Shanghai, China), and hair around the surgical area was shaved and washed. The operative area was then sterilized with iodine (GE Healthcare Life Sciences, Logan, UT, USA), after which an incision into the skin and articular cavity of the medial side of the right knee joint was made. The patella was turned inside-out, with the knee flexed as to expose the articular cavity and enable the anterior and posterior cruciate ligaments to be cut. The above steps were conducted with care to ensure there was no injury to the cartilage. Finally, the articular capsules and the skin were sutured closed subsequent to washing of the joint with normal saline, then the wounds were cleaned with iodine. Each rat in this group received an intramuscular injection of 200,000 U penicillin (GE Healthcare Life Sciences) for anti-inflammation. Subsequent to 7 days of wound healing, the rats in the injured exercise-induced OA model and normal exercise-induced OA model groups underwent training with the animal experimental treadmill to establish the exercise-induced OA model. The first week involved the rats adapting to the treadmill and the exercise load; the exercise intensity was gradually increased (the speed of treadmill was gradually increased from $10 \mathrm{~m} / \mathrm{min}$ to $16 \mathrm{~m} / \mathrm{min}$ ). The formal training commenced in the second week; the speed of the treadmill was maintained at $16 \mathrm{~m} / \mathrm{min}$ and the exercise duration was $30 \mathrm{~min}$. Training lasted two weeks, and each week there were six training days and one rest day.

Tissue preparation and cell culture. Subsequent to establishment of the exercise-induced OA model, the rats were anesthetized by intraperitoneal injection with $10 \%$ chloral hydrate $(0.3 \mathrm{ml} / 100 \mathrm{~g})$, prior to being sacrificed by decapitation. The right knee cartilage of each rat was collected. Samples were sequentially digested by trypsin $(0.5 \%)$ and type II collagenase ( $0.2 \%$; both GE Healthcare Life Sciences). Cells were seeded at a density of $1.5 \times 10^{4}$ cells $/ \mathrm{cm}^{2}$ in Dulbecco's modified Eagle's medium/F12 with $10 \%$ fetal bovine serum in $25 \mathrm{~cm}^{2}$ culture flasks and were cultured until they reached confluence.

$R T-q P C R$. In order to determine the mRNA expression levels of catenin (cadherin-associated protein) $\beta 1$ (Ctnnb1), bone morphogenetic protein 2 (BMP-2), sex determining region Y-box 9 (Sox-9) and runt-related transcription factor 2 (Runx-2) in three groups of chondrocytes, RT-qPCR was conducted. Total RNA was isolated from chondrocytes using TRIzol reagent (Baosheng Biology Co., Ltd., Dalian, China), and mRNA ( $0.1 \mu \mathrm{g})$ was converted to cDNA using the RevertAid ${ }^{\mathrm{TM}}$ First Strand cDNA Synthesis kit (Thermo Fisher Scientific, Inc., Waltham, MA, USA) according to the manufacturer's protocol. Primer sequences are listed in Table I. All primer and probe sets were supplied by TaqMan ${ }^{\circledR}$ Gene Expression Assays (Applied Biosystems; Thermo Fisher Scientific, Inc.). qPCR was performed using $2.0 \mu \mathrm{l} \mathrm{cDNA}$, the SYBR $^{\circledast}$ Premix Ex Taq ${ }^{\mathrm{TM}}$ II kit (Takara Bio, Inc., Otsu, Japan) and the FTC 3000 Real-Time PCR System (Funglyn Biotech Inc., Toronto, ON, Canada). The PCR cycling conditions were as follows: $95^{\circ} \mathrm{C}$ for $30 \mathrm{sec}$, followed by 40 cycles of $95^{\circ} \mathrm{C}$ for $5 \mathrm{sec}$ and $60^{\circ} \mathrm{C}$ for $30 \mathrm{sec}$. The relative fold-change for each individual gene, as normalized to the reference gene ( $\beta$-actin), was calculated using the $2^{-\Delta \Delta C a}$ method (10). The PCR results were analyzed using the Bio-Rad iQ5 software, version 2.1 (Bio-Rad Laboratories, Inc., Hercules, CA, USA). All assays were performed in triplicate.

Western blot analysis. Proteins were extracted from chondrocytes using the Tissue Protein Extraction kit (Thermo Fisher Scientific, Inc.), according to the manufacturer's protocol. The concentration of total protein was quantified using the NanoDrop ND-1000 (Thermo Fisher Scientific, Inc., Wilmington, DE, USA). A total of $12 \mu \mathrm{l}$ total protein was loaded per lane and electrophoresed in $12 \%$ sodium dodecyl sulfate-polyacrylimide gel electrophoresis using a constant voltage of $80 \mathrm{~V}$ for $2 \mathrm{~h}$. The proteins were then transferred onto a 0.45-nitrocellulose membrane (Hybond-ECL; GE Healthcare Life Sciences) at $350 \mathrm{~mA}$ for $90 \mathrm{~min}$ and blocked with $5 \%$ nonfat milk at room temperature for $2 \mathrm{~h}$. The membranes were incubated overnight at $4^{\circ} \mathrm{C}$ with mouse anti-rat collagen II monoclonal antibody (mAb; 1:500; hm1062; Abgent, Inc., San Diego, CA, USA), mouse anti-rat Wnt-3a polyclonal antibody (1:50; 2391S; Cell Signaling Technology, Inc., Danvers, MA, USA), mouse anti-rat $\beta$-catenin mAb (1:50; sc-59737; Santa Cruz Biotechnology, Inc., Dallas, TX, USA), mouse 


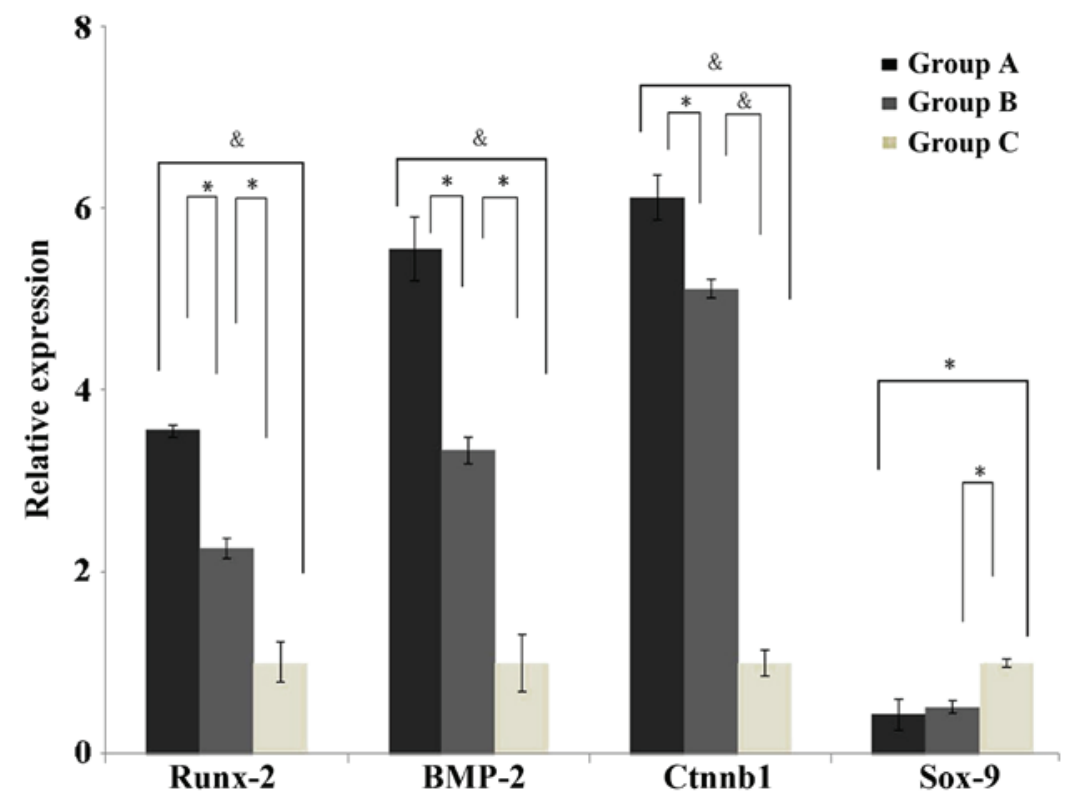

Figure 1. mRNA expression levels of Runx-2, BMP-2, Ctnnb1 and Sox-9 in groups A, B and C. " $\mathrm{P}<0.05$ and ${ }^{\circledR} \mathrm{P}<0.01$ between groups. Runx-2, runt-related transcription factor 2; BMP-2, bone morphogenetic protein 2; Ctnnb1, catenin (cadherin-associated protein) $\beta 1$, Sox-9, sex determining region Y-box 9; group A, injured exercise-induced osteoarthritis group; group B, normal exercise-induced osteoarthritis group; group C, control group.

anti-rat Mmp-13 polyclonal antibody (1:50; bs668; Bioworld Technology, Inc., St. Louis Park, MN, USA) and mouse anti-rat $\beta$-actin monoclonal antibody (1:3,000; CW0096; Abgent, Inc.). Subsequently the membranes were washed three times with Tris-buffered saline containing 5\% Tween-20 (Sinopharm Chemical Reagent Co., Ltd.) for $5 \mathrm{~min}$ each, prior to incubation with horseradish peroxidase-conjugated goat anti-mouse polyclonal IgG (1:4,000; bs-0296p; BIOSS, Beijing, China) at $4^{\circ} \mathrm{C}$ for $2 \mathrm{~h}$, immunoreactive proteins were visualized on an X-ray film (Sinopharm Chemical Reagent Co., Ltd.,) with an Enhanced Chemiluminescence kit (NEN Life Science Products, Inc., Boston, MA, USA). Quantification of band intensity was conducted using Gel-Pro Analyzer 4.0 (Media Cybernetics, Inc., Rockville, MD, USA).

Immunohistochemical staining of Wnt-3a and $\beta$-catenin. Immunohistochemical staining was performed to investigate the expression of key proteins involved in the canonical $\mathrm{Wnt} / \beta$-catenin pathway (i.e. Wnt-3a and $\beta$-catenin) in knee cartilage from rats in the normal exercise-induced OA, injured exercise-induced OA and control groups. Paraffin-embedded (Sinopharm Chemical Reagent Co., Ltd.) 5- $\mu \mathrm{m}$ cartilage sections were prepared using a histotome (Sinopharm Chemical Reagent Co., Ltd.), and were then deparaffinized in xylene (Sinopharm Chemical Reagent Co., Ltd.) and rehydrated in a graded ethanol series and water. Sections were treated with 3\% hydrogen peroxide (Beijing Zhongshan Jinqiao Biotechnology Co.,Ltd., Beijing, China) for $10 \mathrm{~min}$, washed with phosphate-buffered saline (PBS; Sinopharm Chemical Reagent Co., Ltd.) and incubated in $10 \mathrm{M}$ urea solution (GE Healthcare Life Sciences) and trypsin at $37^{\circ} \mathrm{C}$ for 20 min to unmask the antigens. Subsequent to blocking with $5 \%$ goat serum (Beijing Zhongshan Jinqiao Biotechnology Co., Ltd.) for $20 \mathrm{~min}$ at room temperature, mouse polyclonal Wnt-3a and mouse monoclonal $\beta$-catenin antibody (1:50 dilution), as well as the anti-mouse $\operatorname{IgG}(1: 4,000)$ antibody, were applied and incubated overnight at $4^{\circ} \mathrm{C}$. Subsequent to washing with PBS, sections were incubated using the SAP kit (Beijing Zhongshan Jinqiao Biotechnology Co., Ltd.). The substrate 3,3'-diaminobenzidine (Sinopharm Chemical Reagent Co., Ltd.) was added to stain sections with hematoxylin (Sinopharm Chemical Reagent Co., Ltd.) counterstaining. Finally, sections were dehydrated and mounted under coverslips. Wnt-3a and $\beta$-catenin localization in each cartilage zone was assessed systematically by counting the number of positive cells under the SZX16 Stereo Microscope (Olympus Corporation, Tokyo, Japan).

Statistical analysis. Data were presented as the mean \pm standard deviation, and tested for normality and equal variance. Statistical analysis was performed using SPSS software, version 18.0 (SPSS, Inc., Chicago, IL, USA). Differences in the means were determined by one-way analysis of variance for multiple comparisons followed by the least significant difference test for two-group comparisons with multiple comparisons. The Student's t-test was applied to determine differences between two groups. The normality and homogeneity of variance of data were tested prior to statistical analysis. The nonparametric test (Kruskal Wallis) was used when the conditions for normality were not fulfilled. $\mathrm{P}<0.05$ was considered to indicate a statistically significant difference.

\section{Results}

Expression of Runx-2, BMP-2, Ctnnbl and Sox-9 mRNA in the cartilage of the three groups. The mRNA expression levels of Runx-2, BMP-2, Ctnnb1 and Sox-9 were detected using RT-qPCR. As presented in Fig. 1, the results demonstrated that the mRNA expression levels of Runx-2, BMP-2 and Ctnnb1 were significantly increased in the injured exercise-induced OA model and the normal exercise-induced OA model groups, as compared with the control group. In addition, the mRNA expression levels of Runx-2, BMP-2 and Ctnnb1 were significantly 
Table I. Primers used in reverse transcription-quantitative polymerase chain reaction.

\begin{tabular}{llll}
\hline Name & \multicolumn{1}{c}{ 5' Primer sequence } & 3' Primer sequence & Amplicon size (bp) \\
\hline Runx-2 & GCCCAGGCGTATTTCAGATG & GGTAAAGGTGGCTGGGTAGT & 193 \\
BMP-2 & GCAAGAACAAAGCAGGACCA & TGCTTCTTTATGAGGGCCCA & 212 \\
Ctnnb1 & AGACAGCTCGTTGTACTGCT & GTGTCGTGATGGCGTAGAAC & 150 \\
Sox-9 & CAAGAACAAGCCACACGTCA & GTGGTCTTTCTTGTGCTGCA & 141 \\
$\beta$-Actin & CGGAGTCAACGGATTTGGTCGTAT & AGCCTTCTCCATGGTGGTGAAGAC & 120 \\
\hline
\end{tabular}

Runx-2, runt-related transcription factor 2; BMP-2, bone morphogenetic protein 2; Ctnnb1, catenin (cadherin-associated protein) $\beta 1$, Sox-9, sex determining region Y-box 9; bp, base pairs.

Table II. Protein expression levels of chondrocytes in three groups, as measured by western blotting.

\begin{tabular}{lcrr}
\hline \multirow{2}{*}{ Protein } & \multicolumn{3}{c}{ Expression in chondrocytes (densitometry, X/ $\beta$-actin) } \\
\cline { 2 - 4 } Collagen II & Group A & Group B & Group C \\
Mmp-13 & $0.34(0.25-0.43)$ & $0.56(0.42-0.71)$ & $0.68(0.27-1.09)$ \\
$\beta$-Catenin & $1.92(1.49-2.34)$ & $1.35(1.12-1.57)$ & $0.74(0.54-1.15)$ \\
Wnt-3a & $0.95(0.53-1.37)$ & $0.89(0.78-1.00)$ & $0.47(0.22-0.73)$ \\
\hline
\end{tabular}

Group A, injured exercise-induced osteoarthritis group; group B, normal exercise-induced osteoarthritis group; group C, control group; Mmp-13, matrix metalloproteinase 13; Wnt-3a, wingless-type MMTV integration site family member 3A.

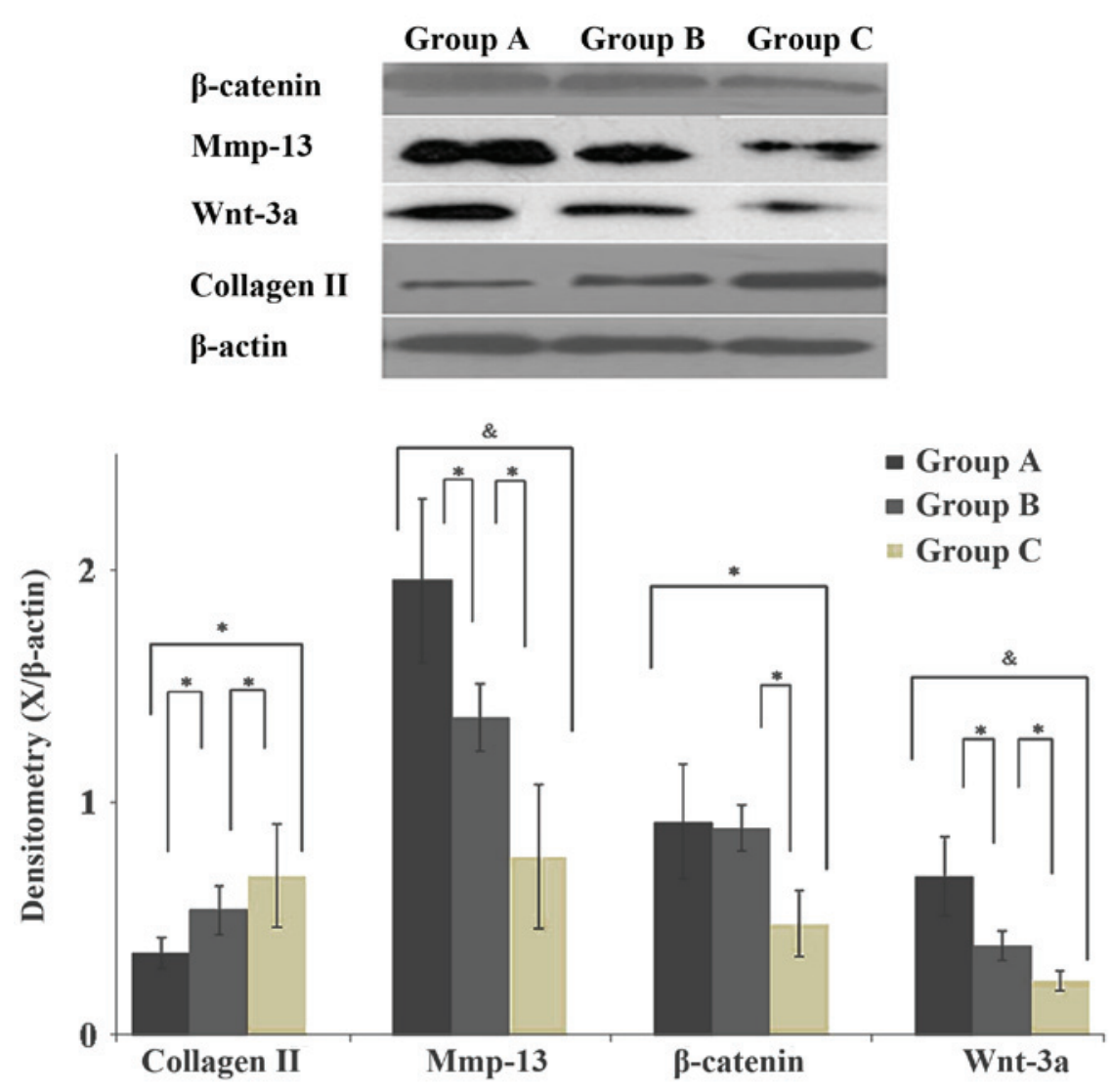

Figure 2. Immunoblotting of $\beta$-catenin, Mmp-13, Wnt-3a and collagen II. The representative immunoblotting images of $\beta$-catenin, Mmp-13, Wnt-3a and collagen II and the densitometric analysis are presented ( $\mathrm{n}=10 /$ group). " $\mathrm{P}<0.05$ and ${ }^{\circledR} \mathrm{P}<0.01$ between groups. Mmp-13, matrix metalloproteinase 13 ; Wnt- $3 \mathrm{a}$, wingless-type MMTV integration site family member 3A; group A, injured exercise-induced osteoarthritis group; group B, normal exercise-induced osteoarthritis group; group C, control group. 
Table III. Protein expression in chondrocytes in the three groups, as determined using immunohistochemistry.

\begin{tabular}{lccr}
\hline & \multicolumn{3}{c}{ Expression in chondrocytes (positive cell rate) } \\
\cline { 2 - 4 } Protein & Group A & Group B & Group C \\
\hline Wnt-3a & $0.62(0.35-0.88)$ & $0.54(0.50-0.58)$ & $0.28(0.17-0.29)$ \\
$\beta-C a t e n i n$ & $0.68(0.38-0.86)$ & $0.50(0.47-0.54)$ & $0.28(0.17-0.29)$
\end{tabular}

Group A, injured exercise-induced osteoarthritis group; group B, normal exercise-induced osteoarthritis group; group C, control group.
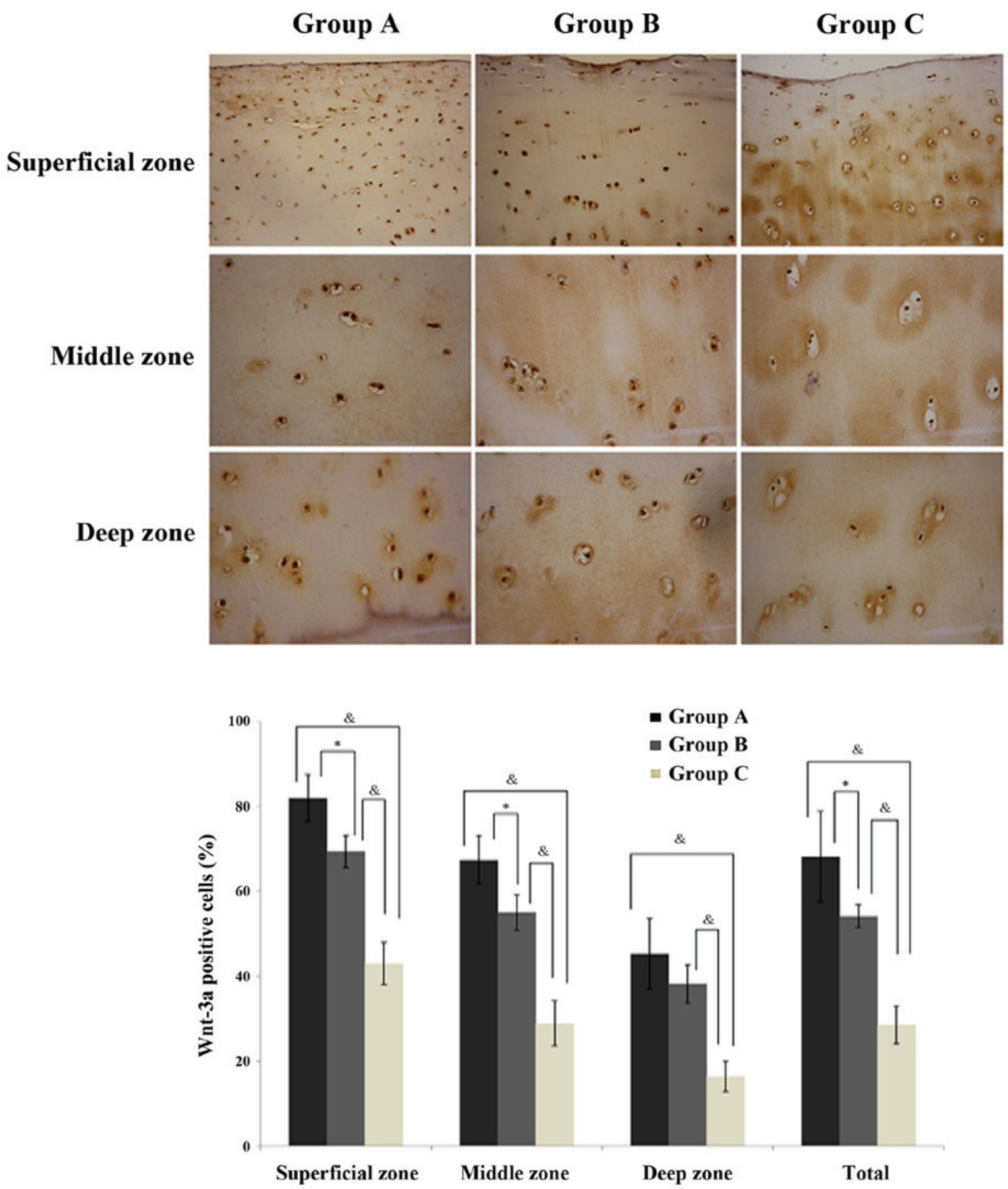

Figure 3. Immunohistochemical localization of Wnt-3a in the chondrocytes ( $\mathrm{n}=10 /$ group). Data are presented as the mean \pm standard deviation. ${ }^{*} \mathrm{P}<0.05$ and ${ }^{\circledR} \mathrm{P}<0.01$ between groups. Magnification of superficial zone, $\mathrm{x} 200$; magnification of middle zone, $\mathrm{x} 400$; and magnification of deep zone, $\mathrm{x} 400$. Wnt-3a, wingless-type MMTV integration site family member 3A; group A, injured exercise-induced osteoarthritis group; group B, normal exercise-induced osteoarthritis group; group C, control group. 

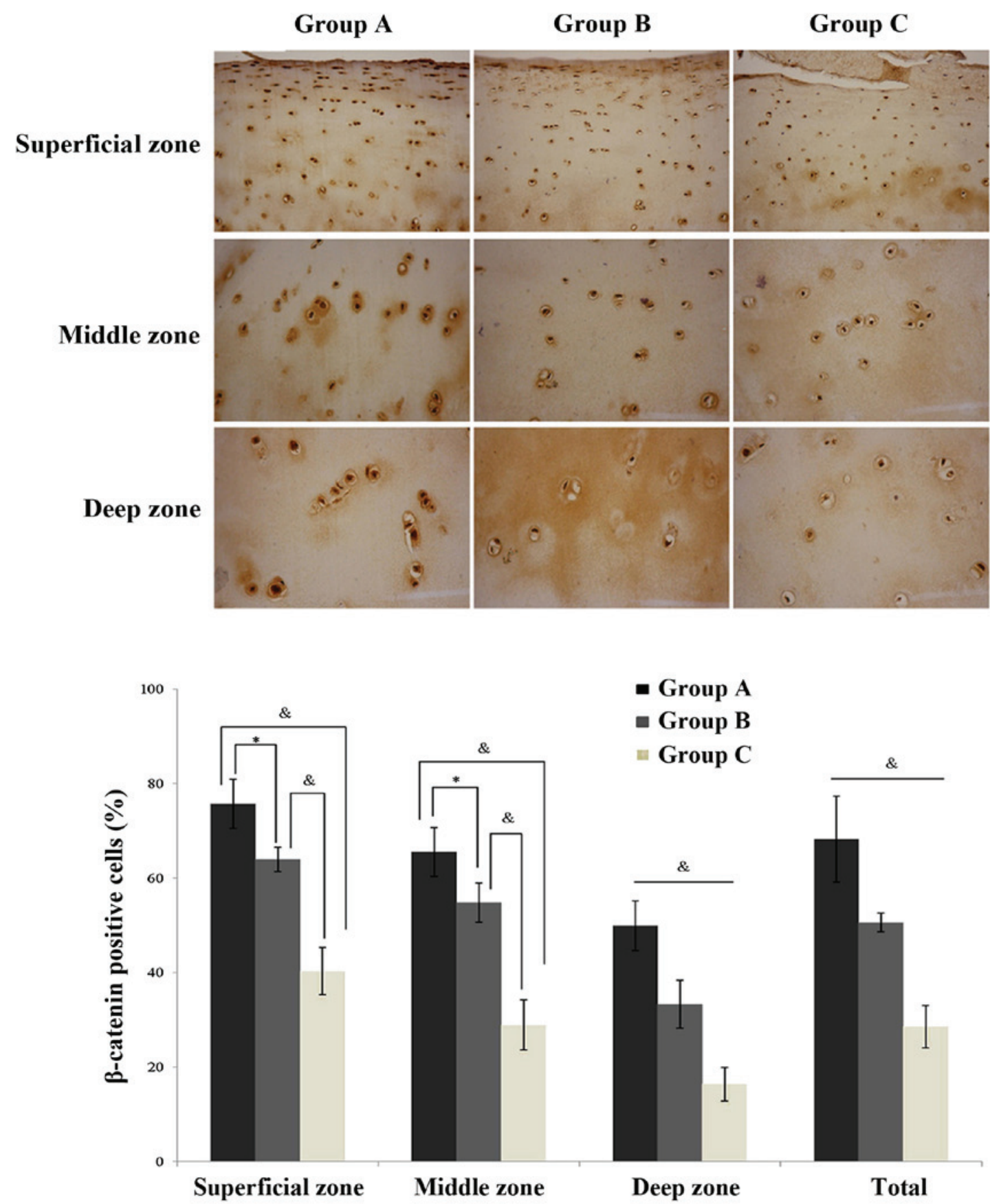

Figure 4. Immunohistochemical localization of $\beta$-catenin in chondrocytes ( $\mathrm{n}=10 /$ group). Data are presented as the mean \pm standard deviation. "P<0.05 and ${ }^{\&} \mathrm{P}<0.01$ between groups. Magnification of superficial zone, $\mathrm{x} 200$; magnification of middle zone, $\mathrm{x} 400$; and magnification of deep zone, $\mathrm{x} 400$. Group A, injured exercise-induced osteoarthritis group; group B, normal exercise-induced osteoarthritis group; group C, control group.

increased in the injured exercise-induced OA model group, as compared with the normal exercise-induced OA model group. Conversely, the mRNA expression levels of Sox-9 were significantly reduced in the injured exercise-induced OA model and the normal exercise-induced OA model groups, as compared with control group. No significant differences were observed between the injured exercise-induced OA model and normal exercise-induced OA model groups in the expression of Sox-9 mRNA.

Expression of collagen II, Mmp-13, Wnt-3a and $\beta$-catenin at the protein level in the three groups. Western blot analysis was conducted to determine the protein expression levels of collagen II, Mmp-13, Wnt-3a and $\beta$-catenin. As presented in Fig. 2, there was a significantly reduced level of collagen II expression in the injured exercise-induced OA model and normal exercise-induced OA model groups, as compared with the control, and expression in the injured exercise-induced OA model group was significantly reduced compared with the normal exercise-induced OA model group $(\mathrm{P}<0.05)$. Wnt-3, $\beta$-catenin, Mmp-13 were all significantly overexpressed in the injured exercise-induced OA model group and the normal exercise-induced OA model groups, as compared with the normal control. In the expression of Wnt-3 and Mmp-13, the 
injured exercise-induced OA model group was observed to exhibit significantly greater expression, as compared with the normal exercise-induced OA model group (Fig. 2; Table II).

Immunostaining of Wnt-3a and $\beta$-catenin. In the cartilage, Wnt-3a (Fig. 3) and $\beta$-catenin (Fig. 4) expression levels were observed to be significantly increased in the normal exercise-induced OA and injured exercise-induced OA groups, as compared with the control group. Additionally, the expression was identified to be significantly greater in the injured exercise-induced OA group, as compared with the normal exercise-induced OA group (Figs. 3 and 4; Table III). The same trends were observed in every zone, a gradual reduction in protein expression in the superficial, middle and deep zones across the injured exercise-induced OA, normal exercise-induced OA and control group cartilages. Thus, it was established that Wnt-3a and $\beta$-catenin are highly expressed in the cartilages of the normal exercise-induced OA and injured exercise-induced OA groups.

\section{Discussion}

OA is a type of chronic arthritis that is characterized by degeneration and deficiency of articular cartilage and hyperostosis of subchondral bone and the edges of joints $(11,12)$. OA is the most common in the knee, and this is representative of OA, manifesting with biochemical and metabolic disturbances and the damage of the articular cartilage structure. Local structural cartilage damage combines inflammation of the synovial membrane and fibroplasia of the joint capsule, which finally lead to pain, swelling and dysfunction of knee joints, potentially resulting in disability (13-15). OA associated with exercise predominantly occurs as a result of excessive abrasiveness and may lead to joint aging and early joint degeneration $(7,16,17)$. The present study aimed to investigate the molecular mechanisms of the key genes and proteins in the canonical Wnt/ $\beta$-catenin pathway in the sport injury-induced $\mathrm{OA}$ by establishing the exercise-induced OA rat model.

Wnt signaling pathway regulates numerous processes, including growth, development, disease, aging and death, cellular morphology and function $(18,19)$. The embryonic development process of higher animals involves cellular proliferation, differentiation, apoptosis and anti-apoptosis, and the $\mathrm{Wnt} / \beta$-catenin pathway is the most well-known pathway in mediating this process (20). $\beta$-catenin is a multifunctional protein which may regulate cell adhesion and signal transduction, and it is the most important component of the Wnt/ $\beta$-catenin pathway $(21,22)$.

Mmps and cytokines serve an important role in the damage of articular cartilage (23), however the mechanisms remain unclear. Previous studies have, however, demonstrated that the $\mathrm{Wnt} / \beta$-catenin pathway regulated the expression levels of Mmps and the BMP gene families (24). Zhu et al (21) identified that the overexpression of $\beta$-catenin may lead to OA-like alterations in the knee joints of mice, and increases in the gene expression of Mmp-9, Mmp-13 and BMP-2. The activity of gelatinase and the gene expression of $\mathrm{Mmp}-3$ and Mmp-13 has been previously identified to be increased subsequent to the activation of the $\mathrm{Wnt} / \beta$-catenin pathway by Wnt-3a in the articular cartilage of rabbit (25). Based on the abovementioned studies, the $\mathrm{Wnt} / \beta$-catenin pathway is suggested to serve a key role in the mechanism of OA. In the present study, the expression of BMP-2 and Mmp-13, the marker genes of chondrocyte maturation and the downstream genes in the Wnt/ $\beta$-catenin pathway (20), were significantly different among the three groups.

$\mathrm{BMP}$ is involved in the regulation of the differentiation, proliferation, maturity and apoptosis and may increase the expression of Runx-2 downstream (26). The BMP content was significantly increased in the synovium and cartilage tissue in the mouse model of OA induced by papain, and it was observed that the BMP inhibitor was able to reverse the formation of osteophytes in OA (27). When the process of endochondral ossification is completed, overexpression of BMP leads to hypertrophy, differentiation of chondrocytes and the formation of osteophytes (28). The overexpression of BMP-2 mRNA has been previously identified in the chondrocytes of OA knees in the patients with mechanical injury (28). A previous study identified that the expression of BMPs and Runx-2 were increased significantly in the peripheral blood of patients with OA, therefore BMPs and Runx-2 may be considered as markers for the activity of disease and reaction to treatment (29). In the current study, Runx-2 and BMP-2 mRNA expression levels were identified to be upregulated, and the Runx-2 and BMP-2 protein expression levels were increased in the injured exercise-induced OA model group and exercise-induced OA model group compared with the control group. This indicated that the normal exercise-induced OA group had similar alterations in gene and protein expression, as compared with the injured exercise-induced OA group; however whether these alterations were induced by the $\mathrm{Wnt} / \beta$-catenin pathway requires further investigation.

Mmp-13 is the most effective collagen II degradation enzyme and the overexpression of Mmp-13 results in damage to the articular cartilage (30). A previous study additionally identified that the high expression levels of Mmp-13 in transgenic mice could result in the appearane of OA-like pathological alterations (30). The Wnt/ $\beta$-catenin signaling pathway was suggested to serve an important role in this process; overexpression of $\beta$-catenin can lead to increases in Mmp-13 gene expression, which contributes to the degradation of cartilage matrix and damage of articular cartilage $(21,31)$.

Sox-9 regulates the development of cartilage in the early phase and sustains the specialty of chondrocytes (32). Sox-9 serves a key regulating role in controlling differentiation and hypertrophy of chondrocytes (32). Additionally, it has been reported that $\mathrm{Wnt} / \beta$-catenin signaling pathway does not serve a role in differentiation and hypertrophy of chondrocytes if the expression of Sox-9 is reduced, indicating that the $\mathrm{Wnt} / \beta$-catenin signaling pathway-mediated promotion of chondrocyte differentiation is dependent on the expression of Sox-9 (32,33). Sox-9 has also been reported to regulate the expression of collagen II, and it is notable that the Wnt/ $\beta$-catenin signaling pathway may regulate the upstream Sox-9 gene as part of a feedback regulation (34). The results of the current study identified that the expression of Sox-9 was reduced, and the expression of $\beta$-catenin was increased at mRNA and protein levels, thus indicating that increased expression levels of $\beta$-catenin regulate Sox-9 via feedback regulation in this exercise-induced OA rat model. 
In the present study, upregulation of $\beta$-catenin and Wnt-3a was identified in the injured exercise-induced OA model and exercise-induced OA model groups. Therefore, $\beta$-catenin and Wnt-3a may participate in the pathogenesis of exercise-induced OA by abnormally activating the Wnt/ $\beta$-catenin pathway as a result of frequently excessive pressure in sports. This may provide an improved understanding of the molecular pathogenesis of exercise-induced OA, thus providing a basis for future research.

\section{Acknowledgements}

The authors would like to thank the Laboratory Animal Center of Xi'an Jiaotong University Health Science Center for their support and cooperation in the collection of animals and the experimental methods. They would also like to thank Mr. Shen Wan, Mr. Shuilang He, Miss. Chuiyan Wen, Dr Chunying Liu and Mr. Yang Wen for their technical assistance.

\section{References}

1. Buckwalter JA, Mankin HJ and Grodzinsky AJ: Articular cartilage and osteoarthritis. Instr Course Lect 54: 465-480, 2005.

2. Ramos YF, Bos SD, Lakenberg N, Böhringer S, den Hollander WJ, Kloppenburg M, Slagboom PE and Meulenbelt I: Genes expressed in blood link osteoarthritis with apoptotic pathways. Ann Rheum Dis 73: 1844-1853, 2014.

3. Ren YH: The epidemiological investigation of sports injury in elite athletes. Zhong guo yundong yi xue 19: 377-386, 2000 (In Chinese).

4. Minns RJ and Muckle DS: The role of the meniscus in an instability model for osteoarthritis in the rabbit knee. Br J Exp Pathol 63: 18-24, 1982.

5. Setton LA, Mow VC, Müller FJ, Pita JC and Howell DS Mechanical behavior and biochemical composition of canine knee cartilage following periods of joint disuse and disuse with remobilization. Osteoarthritis Cartilage 5: 1-16, 1997.

6. Buckwalter JA and Martin JA: Sports and osteoarthritis. Curr Opin Rheumatol 16: 634-639, 2004.

7. Lequesne M: Sport practice and osteoarthritis of the limbs. Science \& Sports 19: 281-285, 2004 (In French).

8. Nusse R: Wnt signaling in disease and in development. Cell Res 15: 28-32, 2005.

9. Nelson WJ and Nusse R: Convergence of Wnt, beta-catenin, and cadherin pathways. Science 303: 1483-1487, 2004.

10. Livak KJ and Schmittgen TD: Analysis of relative gene expression data using real-time quantitative PCR and the $2-\Delta \Delta \mathrm{Ct}$ method Methods 25: 402-408, 2001.

11. Aigner T, Rose J, Martin J and Buckwalter J: Aging theories of primary osteoarthritis: From epidemiology to molecular biology. Rejuvenation Res 7: 134-145, 2004.

12. Loeser RF, Goldring SR, Scanzello CR and Goldring MB: Osteoarthritis: A disease of the joint as an organ. Arthritis Rheum 64: 1697-1707, 2012.

13. Benito MJ, Veale DJ, Fitzgerald O, van den Berg WB and Bresnihan B: Synovial tissue inflammation in early and late osteoarthritis. Ann Rheum Dis 64: 1263-1267, 2005.

14. Sellam $J$ and Berenbaum F: The role of synovitis in pathophysiology and clinical symptoms of osteoarthritis. Nat Rev Rheumatol 6: 625-635, 2010.
15. Ma CH, Lv Q, Cao Y, Wang Q, Zhou XK, Ye BW and Yi CQ: Genes relevant with osteoarthritis by comparison gene expression profiles of synovial membrane of osteoarthritis patients at different stages. Eur Rev Med Pharmacol Sci 18: 431-439, 2014.

16. Eckstein F, Hudelmaier M and Putz R: The effects of exercise on human articular cartilage. J Anat 208: 491-512, 2006.

17. Almekinders LC and Garrett WE: Sports injuries. Curr Probl Surg 37: 331-338, 2000.

18. Strutt D: Frizzled signalling and cell polarisation in Drosophila and vertebrates. Development 130: 4501-4513, 2003.

19. Luyten FP, Tylzanowski $P$ and Lories RJ: Wnt signaling and osteoarthritis. Bone 44: 522-527, 2009.

20. Wodarz A and Nusse R: Mechanisms of Wnt signaling in development. Annu Rev Cell Dev Biol 14: 59-88, 1998.

21. Zhu M, Tang D, Wu Q, Hao S, Chen M, Xie C, Rosier RN, O'Keefe RJ, Zuscik M and Chen D: Activation of beta-catenin signaling in articular chondrocytes leads to osteoarthritis-like phenotype in adult beta-catenin conditional activation mice. J Bone Miner Res 24: 12-21, 2009.

22. Piters E, Boudin E and Van Hul W: Wnt signaling: A win for bone. Arch Biochem Biophys 473: 112-116, 2008.

23. Burrage PS, Mix KS and Brinckerhoff CE: Matrix metalloproteinases: Role in arthritis. Front Biosci 11: 529-543, 2006.

24. Kawaguchi H: Regulation of osteoarthritis development by Wnt-beta-catenin signaling through the endochondral ossification process. J Bone Miner Res 24: 8-11, 2009.

25. Yuasa T, Otani T, Koike T, Iwamoto M and Enomoto-Iwamoto M: Wnt $/ \beta$-catenin signaling stimulates matrix catabolic genes and activity in articular chondrocytes: Its possible role in joint degeneration. Lab Invest 88: 264-274, 2008.

26. Guohui Y: Bone morphogenetic protein in the repair of articular cartilage injury. J Clin Rehabil Tissue Eng Res 46: 8669-8672, 2010.

27. Scharstuhl A, Vitters EL, van der Kraan PM and van den Berg WB: Reduction of osteophyte formation and synovial thickening by adenoviral overexpression of transforming growth factor beta/bone morphogenetic protein inhibitors during experimental osteoarthritis. Arthritis Rheum 48: 3442-3451, 2003.

28. Dell'Accio F, De Bari C, El Tawil NM, Barone F, Mitsiadis TA, O'Dowd J and Pitzalis C: Activation of WNT and BMP signaling in adult human articular cartilage following mechanical injury. Arthritis Res Ther 8: R139, 2006.

29. Grcevic D, Jajic Z, Kovacic N, Lukic IK, Velagic V, Grubisic F, Ivcevic $\mathrm{S}$ and Marusic A: Peripheral blood expression profiles of bone morphogenetic proteins, tumor necrosis factor-superfamily molecules and transcription factor Runx 2 could be used as markers of the form of arthritis, disease activity, and therapeutic responsiveness. J Rheumatol 37: 246-256, 2010.

30. Neuhold LA, Killar L, Zhao W, Sung ML, Warner L, Kulik J, Turner J, Wu W, Billinghurst C, Meijers T, et al: Postnatal expression in hyaline cartilage of constitutively active human collagenase-3 (MMP-13) induces osteoarthritis in mice. J Clin Invest 107: 35-44, 2001.

31. Nakashima A and Tamura M: Regulation of matrix metalloproteinase-13 and tissue inhibitor of matrix metalloproteinase-1 gene expression by WNT3A and bone morphogenetic protein-2 in osteoblastic differentiation. Front Biosci 11: 1667-1678, 2006.

32. Topol L, Chen W, Song H, Day TF and Yang Y: Sox9 inhibits Wnt signaling by promoting beta-catenin phosphorylation in the nucleus. J Biol Chem 284: 3323-3333, 2009.

33. Hwang SG, Yu SS, Lee SW and Chun JS: Wnt-3a regulates chondrocyte differentiation via c-Jun/AP-1 pathway. FEBS Lett 579: 4837-4842, 2005.

34. Miclea RL, Karperien M, Bosch CA, van der Horst G, van der Valk MA, Kobayashi T, Kronenberg HM, Rawadi G, Akçakaya $\mathrm{P}$, Löwik CW, et al: Adenomatous polyposis coli-mediated control of beta-catenin is essential for both chondrogenic and osteogenic differentiation of skeletal precursors. BMC Dev Biol 9: 26, 2009. 